\title{
Aerobic biological treatment of waste- waters containing dichloromethane
}

\author{
Sandra C Moura, ${ }^{1}$ Ruben Ferreira Jorge, ${ }^{1}$ Anouk Duque, ${ }^{1}$ Rui AR Boaventura ${ }^{2}$ and \\ Paula ML Castro ${ }^{1 *}$ \\ ${ }^{1}$ Escola Superior de Biotecnologia, Universidade Católica Portuguesa, R. Dr. António Bernardino de Almeida, 4200-072 Porto, Portugal \\ ${ }^{2}$ LSRE - Departamento de Engenharia Química, Faculdade de Engenharia da Universidade do Porto (FEUP), R. Dr. Roberto Frias, \\ 4200-465 Porto, Portugal
}

\begin{abstract}
BACKGROUND: Volatilization has been advanced as one of the predominant phenomena contributing to volatile organic carbon emissions from wastewater treatment plants (WWTPs). In this study, strategies for minimizing such air stripping losses when treating a liquid stream containing dichloromethane (DCM), aiming at decreasing the overall emission inventory from WWTPs, were investigated.

RESULTS: System R1, consisting of a continuous flow stirred tank reactor (CSTR) treating a liquid stream containing DCM at a concentration of $12 \mathrm{mmol} \mathrm{dm} \mathrm{dm}^{-3}$ presented a biodegradation efficiency (BE) of $68 \%$, based upon chloride release, with $10 \%$ of measurable losses, mainly due to volatilization, and $22 \%$ of unmeasurable losses. System R2 introduced operational designs aiming at decreasing DCM volatilization. In Experiment R2.1, a biotrickling filter, through which the air stripped from the CSTR was driven, was introduced leading to a reduction from $10 \%$ to $7 \%$ on the measurable losses. In Experiment R2.2, the air stripped from the CSTR was recirculated at a flow rate of $2.4 \mathrm{dm}^{3} \mathrm{~h}^{-1}$ through the reactor medium before entering the biotrickling filter. The BE was improved from $69 \%$ to $82 \%$ and the losses associated with air stripping were successfully reduced to $2 \%$. The proposed design, including air recirculation and the biotrickling filter, increased the ratio between the biodegradation rate and the volatilization rate from 7 to 41 .
\end{abstract}

CONCLUSIONS: Recirculation of the gaseous effluent through the reactor medium, which allowed for higher residence time within the bioreactor, was shown to be a successful strategy for improving the treatment process, thus minimizing DCM volatilization losses.

Keywords: dichloromethane; biodegradation; volatilization losses; VOC emissions

\section{INTRODUCTION}

Emissions of non-methane volatile organic compounds (NMVOCs) are increasingly under legislative control since they are associated with a variety of environmental impacts at local, regional and global scales. Volatilization has been advanced as one of the predominant phenomena contributing to NMVOCs emissions from wastewater treatment plants (WWTPs) via three main mechanisms: diffusive volatilization, evaporation and convective stripping. ${ }^{1-3}$

Diffusive volatilization and evaporation mechanisms are driven by atmospheric conditions, namely wind and temperature, respectively. Regarding convective stripping, mechanical agitation and/or forced-air flow are the main factors involved. Air stripping is commonly found in aerated WWTPs and aerated transfer channels, ${ }^{1,4}$ and is more significant when non-contaminated air is used in the aeration of the wastewater. Emissions of NMVOCs from WWTPs have previously been characterized. ${ }^{1-3}$ Theoretical models have been developed to characterize the importance of volatilization relative to other transport processes under varying environmental conditions. Estimation of NMVOC emission rates based on the stagnant film model showed that the aerated biological treatment unit had the highest contribution $(>80 \%)$ of total NMVOCs emissions from WWTPs using forced aeration. ${ }^{3}$ Limited data is available on the removal of NMVOC emissions from WWTPs. ${ }^{5}$

Novel bioreactor designs, namely membrane bioreactors, have been proposed as effective technologies to minimize air stripping losses associated with wastewater treatment. ${ }^{6,7}$ Other studies proposed the application of membrane aeration bioreactors, in which oxygen is transferred using a synthetic membrane to provide a bubbleless aeration for WWTPs, as a way of minimizing these phenomena. ${ }^{8}$ Other options involve the use of hydrogen peroxide as the oxygen source,

* Correspondence to: Paula ML Castro, Escola Superior de Biotecnologia - Universidade Católica Portuguesa, R. Dr. António Bernardino de Almeida, $4200-072$ Porto, Portugal 
thus eliminating the need for aeration. ${ }^{6}$ However, no studies to date have been carried out into the operation of a WWTP treating wastewater containing NMVOCs when convective stripping is used for aeration.

Dichloromethane (DCM) is one of the major solvents consumed by the pharmaceutical industry and its use can cause emissions to air, water resources and land. DCM biodegradability was first reported in $1978 .{ }^{9}$ Due to its high vapour pressure, DCM can be partly volatilized from aerated tanks during treatment of waste effluents in WWTPs. ${ }^{10}$ The relative importance of the two main processes occurring in WWTPs, namely biodegradation and air stripping, has been reported to be a factor of $12 .^{11}$ The present study aimed at investigating the aerobic biological treatment of a liquid stream containing DCM with the use of forced air for aeration. Operational strategies for minimization of air stripping losses were devised, aimed at the development of control strategies capable of decreasing the overall emission inventory from WWTPs.

\section{MATERIAL AND METHODS}

\section{Inoculum source}

The inoculum used in these experiments was a mixed culture previously enriched in the laboratory from soil samples collected in the vicinity of an industrial state in the centre of Portugal, which is able to use DCM as a sole source of carbon and energy (unpublished data). The composition of the mineral medium used in these experiments has been described elsewhere. ${ }^{12}$ Microbial cultures were maintained on slopes of nutrient agar $(15 \% \mathrm{w} / \mathrm{w})$ at $5{ }^{\circ} \mathrm{C}$ and subcultured when required. Culture flasks were incubated at $25^{\circ} \mathrm{C}$ with gentle agitation with DCM concentrations of $1.2 \mathrm{mmol} \mathrm{dm}^{-3}$. The bioreactor was inoculated with $0.5 \mathrm{dm}^{3}$ of the degrading culture at a biomass concentration of $10 \mathrm{mg} \mathrm{dm}^{-3}$.

\section{Reactor design and operation}

The reactor used in the experiments was operated as a continuous flow stirred tank reactor (CSTR) with a working volume of $1.5 \mathrm{dm}^{3}$. The experimental set-up used is shown in Fig. 1. The reactor was operated for the treatment of a liquid stream composed of mineral medium containing DCM at $12 \mathrm{mmol} \mathrm{dm}^{-3}$, unless otherwise stated, fed at a flow rate of $0.1 \mathrm{dm}^{3} \mathrm{~h}^{-1}$. The $\mathrm{pH}$ was automatically controlled at $7 \pm 0.5$ and the bioreactor was continuously stirred at $200 \mathrm{rpm}$. The oxygen required for complete aerobic mineralization of the DCM was estimated from the organic load fed into the reactor, according to the complete aerobic degradation of DCM into carbon dioxide and biomass. Upon setting the operational parameters, the overall mass-transfer coefficient ( $\mathrm{kLa})\left(20^{\circ} \mathrm{C}\right)$ was determined to be $0.651 \mathrm{~h}^{-1}$, following a transient aeration method in the absence of microorganisms, and the required air flow was set at $8.2 \mathrm{dm}^{3} \mathrm{~h}^{-1}(0.091$ vvm [air volume per liquid media volume per minute]), assuming a mean concentration of dissolved oxygen in the medium of $3 \mathrm{mg} \mathrm{dm}^{-3}$.

A series of different experiments was carried out. Experiment R1 consisted of a CSTR treating a liquid stream of DCM and its performance was characterized. Experiment R2 introduced operating strategies aimed at improving the treatment performance. In Experiment R2.1, a $0.1 \mathrm{dm}^{3}$ biotrickling column was connected to the bioreactor and was fed, in an upflow mode, with the outlet gas from the bioreactor.

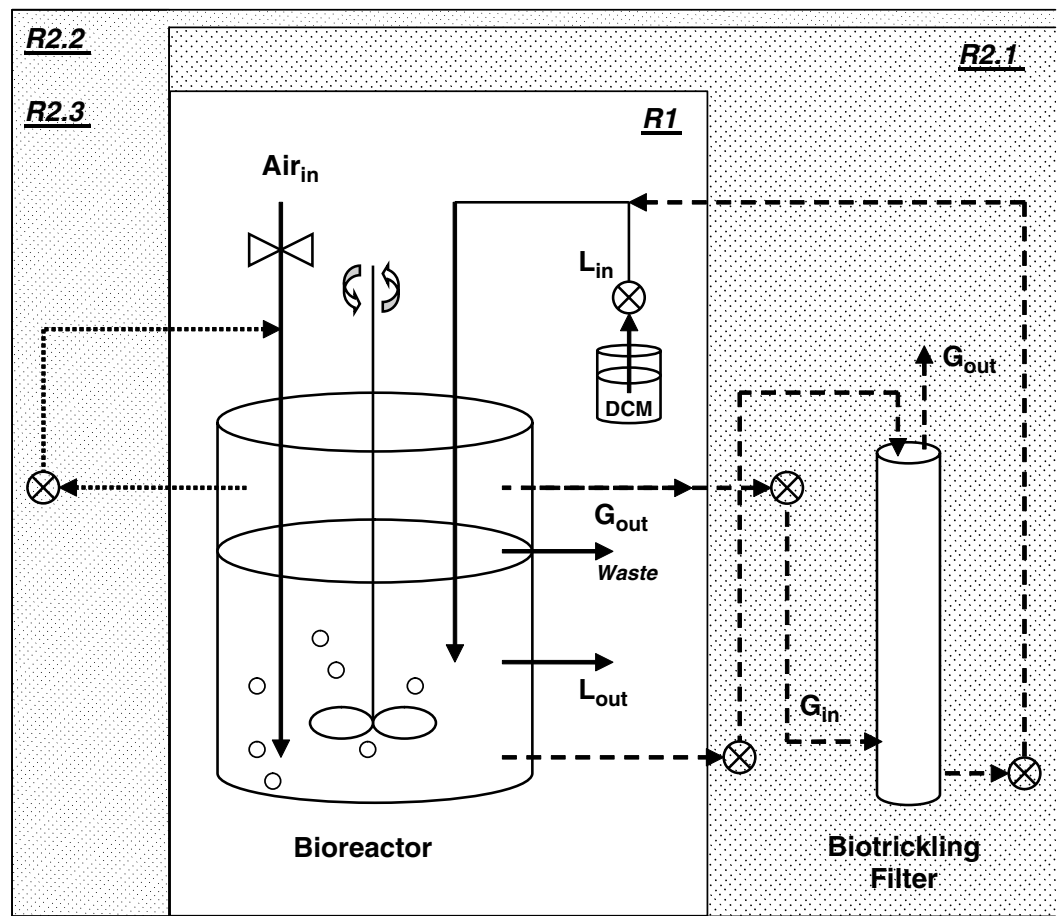

Figure 1. Schematic representation of the various set-ups used in the experiments carried out on System R1 and System R2. 
Liquid medium from the bioreactor was circulated in counter-current to the waste gas at $1.5 \mathrm{dm}^{3} \mathrm{~h}^{-1}$. The biotrickling filter was packed with PVC Raschig rings. Finally, in Experiments R2.2 and R2.3 recirculation of the waste gas back to the bioreactor medium, before entering the biotrickling filter, was introduced. The gas recirculation flow was set at $2.4 \mathrm{dm}^{3} \mathrm{~h}^{-1}(0.025 \mathrm{vvm})$ and $3.6 \mathrm{dm}^{3} \mathrm{~h}^{-1}$ (0.037 vvm), respectively. DCM was monitored at the bioreactor inlet and outlet of both liquid and gaseous streams. The liquid streams were also monitored for quantification of suspended biomass and chloride concentration. Experiments R2 were carried out sequentially.

Mass balances on each system were carried out aimed at quantifying the relative importance of biodegradation and the air stripping phenomena during the treatment process. The balance was carried out after each system reached a pseudo-steady state, determined by constancy in the chloride levels, and involving quantification of the organic load, the biological elimination capacity (BEC) and the measurable losses. The BEC was calculated from the chloride evolution and was expressed in $\mathrm{mol} \mathrm{m}^{-3}$ system $\mathrm{d}^{-1}$. Measurable losses included losses from volatilization of DCM and losses of DCM in the outlet liquid stream. Volatilization losses were quantified at the outlet stream of each system, i.e. bioreactor outlet at System R1 and biotrickling filter outlet at System $\mathrm{R} 2$, and expressed as volatilization rate $\left(\mathrm{VR}, \mathrm{mol} \mathrm{m}^{-3}\right.$ system $\left.\mathrm{d}^{-1}\right)$. Liquid losses of DCM were quantified at the outlet liquid stream. Other losses, which included adsorption onto system parts and gaseous losses at the outlet liquid stream, were estimated globally from the mass balance, and are referred to in this study as unmeasurable losses. The performance of each system was also evaluated in terms of the biodegradation efficiency (BE, \%), calculated from the BEC and the total DCM fed to the system.

\section{Chemicals}

All chemicals used in preparation of media were obtained from $\mathrm{BDH}$ and were of AnalaR grade. All the organic solvents were obtained from Sigma-Aldrich Chemie (Germany).

\section{Analytical Methods}

Chloride concentration was assayed colorimetrically by the mercury thiocyanate method. ${ }^{13}$ For sample preparation, biomass was previously removed by centrifugation at $10000 \mathrm{rpm}$ for $10 \mathrm{~min}$. Chloride concentration was calculated from a standard calibration curve. The uncertainty in this assay (quoted as the standard error of five separate samples, calculated by dividing the standard deviation by the mean of the samples) was $\pm 5 \%$ at the $10 \mathrm{mmol} \mathrm{dm}^{-3}$ level. Biomass concentration was monitored by measuring the absorbance at $600 \mathrm{~nm}$ and calculated from a previously established dry-weight calibration curve. Oxygen concentration measurements were carried out using an oxygen probe (Ingold, UK).
Concentration of DCM present in the liquid phase was determined by gas chromatography via standard calibration curves. Standard solutions were prepared on aqueous basis and allowed to homogenize for $12 \mathrm{~h}$. $4.5 \mathrm{~cm}^{3}$ of each standard solution or sample were extracted with $2 \mathrm{~cm}^{3}$ of diethyl ether for $5 \mathrm{~min}$ and $1 \mathrm{~mm}^{3}$ of the extract was injected into a gas chromatograph (Hewlett Packard 5890, UK) equipped with a flame ionization detector, a CP-wax column $52 \mathrm{CB}$ $(25 \mathrm{~m} \times 0.25 \mathrm{~mm} \times 0.2 \mu \mathrm{m})$ fused silica (SGE, Australia) and using hydrogen (flow $1.2 \mathrm{~cm}^{3} \mathrm{~min}^{-1}$ ) as the carrier gas. The gas chromatograph was operated using the following conditions: injector temperature, $220^{\circ} \mathrm{C}$; oven temperature initially set at $50^{\circ} \mathrm{C}$ for $2 \mathrm{~min}$ then increased at a rate of $25^{\circ} \mathrm{C} \mathrm{min}^{-1}$ to $150^{\circ} \mathrm{C}$, followed by another increase at $50^{\circ} \mathrm{C} \mathrm{min}^{-1}$ to $220^{\circ} \mathrm{C}$; splitless, $30 \mathrm{~s}$; split flow, $60 \mathrm{~cm}^{3} \mathrm{~min}^{-1}$. All samples were analysed at least twice. Calibration was performed weekly. The uncertainty of the assay was $\pm 5 \%$ at DCM concentrations of $10 \mathrm{mmol} \mathrm{dm}^{-3}$.

\section{RESULTS AND DISCUSSION Experiment R1}

To quantify DCM losses, a $1 \mathrm{dm}^{3}$ flask containing mineral medium (MM) with $12 \mathrm{mmol} \mathrm{dm}^{-3} \mathrm{DCM}$ was stripped with a stream of air at $8.2 \mathrm{dm}^{3} \mathrm{~h}^{-1}$, using a single airstone sparger in the absence of stirring. After $1.5 \mathrm{~h}, \mathrm{DCM}$ concentration was reduced to half and after $6 \mathrm{~h}$ DCM was no longer detected in the liquid phase. This result emphasizes the potential significance of air stripping phenomenon when considering the biological treatment of this pollutant in a WWTP due to the high DCM vapour pressure. ${ }^{10}$ This physical property of DCM has been explored aiming at its removal from contaminated groundwater by air stripping. ${ }^{14}$

In Experiment R1, DCM was initially fed at $6 \mathrm{mmol} \mathrm{dm}{ }^{-3}$. Release of chloride was observed from the start of the experiment and stabilized at $7 \mathrm{mmol} \mathrm{dm}^{-3}$ after 6 days of operation (Fig. 2). Gaseous DCM outlet concentrations also stabilized at $5 \mathrm{mmol} \mathrm{m}{ }^{-3}$. On day 10 , the DCM inlet concentration was increased to $12 \mathrm{mmol} \mathrm{dm}^{-3}$ and an increase in both chloride and DCM outlet gaseous concentrations was observed. Chloride concentration stabilized at $15 \mathrm{mmol} \mathrm{dm}^{-3}$, while the DCM gaseous outlet concentration showed a similar pattern to that observed on the DCM inlet feed, with the highest values observed after the DCM inlet feed was renewed. Such fluctuations observed in the inlet DCM concentrations were due to higher mass volatilization with increasing gas volumes in the feed bottle. A low suspended biomass concentration was observed throughout this experiment, which was considered to be mainly due to a stable biofilm formed on the reactor walls. The liquid DCM concentration in the CSTR outlet was negligible throughout the experiment.

A mass balance in Experiment R1, based on chloride release, showed that the BE was $68 \%$, which 


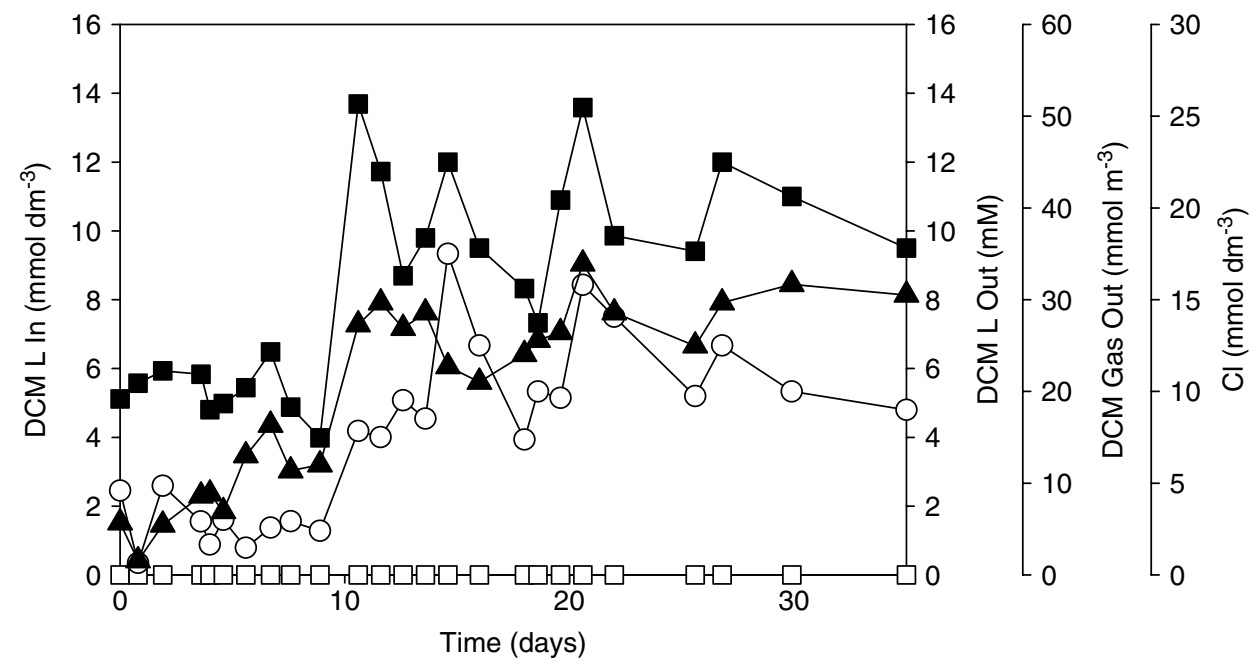

Figure 2. System R1. Symbols: DCM liquid in (घ); DCM liquid out ( $\square$ ); DCM gas out (O); and $\mathrm{Cl}^{-}$

Table 1. Overall treatment performance of System R.1 and System R.2

\begin{tabular}{|c|c|c|c|c|}
\hline & \multirow[b]{2}{*}{$\begin{array}{c}\text { System } \\
\text { R1 }\end{array}$} & \multicolumn{3}{|c|}{ System R2 } \\
\hline & & $\begin{array}{c}\text { Experiment } \\
\text { R2.1 }\end{array}$ & $\begin{array}{l}\text { Experiment } \\
\text { R2.2 }\end{array}$ & $\begin{array}{c}\text { Experiment } \\
\text { R2.3 }\end{array}$ \\
\hline $\begin{array}{l}\text { Biodegradation } \\
\text { rate }(\%)\end{array}$ & 68 & 69 & 82 & 84 \\
\hline $\begin{array}{l}\text { Measurable } \\
\text { losses (\%) }\end{array}$ & 10 & 6.8 & 1.8 & 4.7 \\
\hline $\begin{array}{c}\text { Unmeasurable } \\
\text { losses (\%) }\end{array}$ & 22 & 24.2 & 16.2 & 11.3 \\
\hline $\begin{array}{r}\text { BEC }\left(\mathrm{mol} \mathrm{m}^{-3}\right. \\
\text { system d } \\
-1\end{array}$ & 10.5 & 11.6 & 12.2 & 12.5 \\
\hline BECNR & 7 & 10 & 41 & 17 \\
\hline
\end{tabular}

corresponded to a BEC of $10.5 \mathrm{~mol} \mathrm{~m}^{-3}$ system $\mathrm{d}^{-1}$ (Table 1). Other studies using immobilized cells ${ }^{15}$ and a fluidized bioreactor ${ }^{16}$ gave much higher BEC, namely $463 \mathrm{~mol} \mathrm{~m}^{-3}$ bioreactor $\mathrm{d}^{-1}$ and $146 \mathrm{~mol} \mathrm{~m}^{-3}$ bioreactor $\mathrm{d}^{-1}$, respectively. The lower BEC observed in this study may be explained by the low biomass concentration in the bioreactor when equilibrium was established. The measurable losses, only attributed to DCM volatilization as the DCM concentration in the outlet liquid stream was negligible, were quantified at $0.1 \mathrm{mmolh}^{-1}$ and corresponded to $10 \%$ of the total DCM fed to the system. The unmeasurable losses were quantified as $22 \%$ of the total DCM (Table 1). Overall, the BEC was approximately seven times higher than the VR, a value that is comparable to others reported in the literature, namely a factor of 12 based on model simulations. ${ }^{11}$

\section{Experiment R2}

Upon identifying volatilization from the bioreactor as the main source of DCM measurable losses, operational strategies were investigated in Experiment R2. Experiment R2.1 introduced a biotrickling filter column through which the headspace of the CSTR was treated (Fig. 1). When compared to Experiment $\mathrm{R} 1$, the reactor start-up was slower, as shown by the evolution of chloride in the medium, which did not start until after day 10 (Fig. 3). During this initial period, DCM was quantified at both liquid and

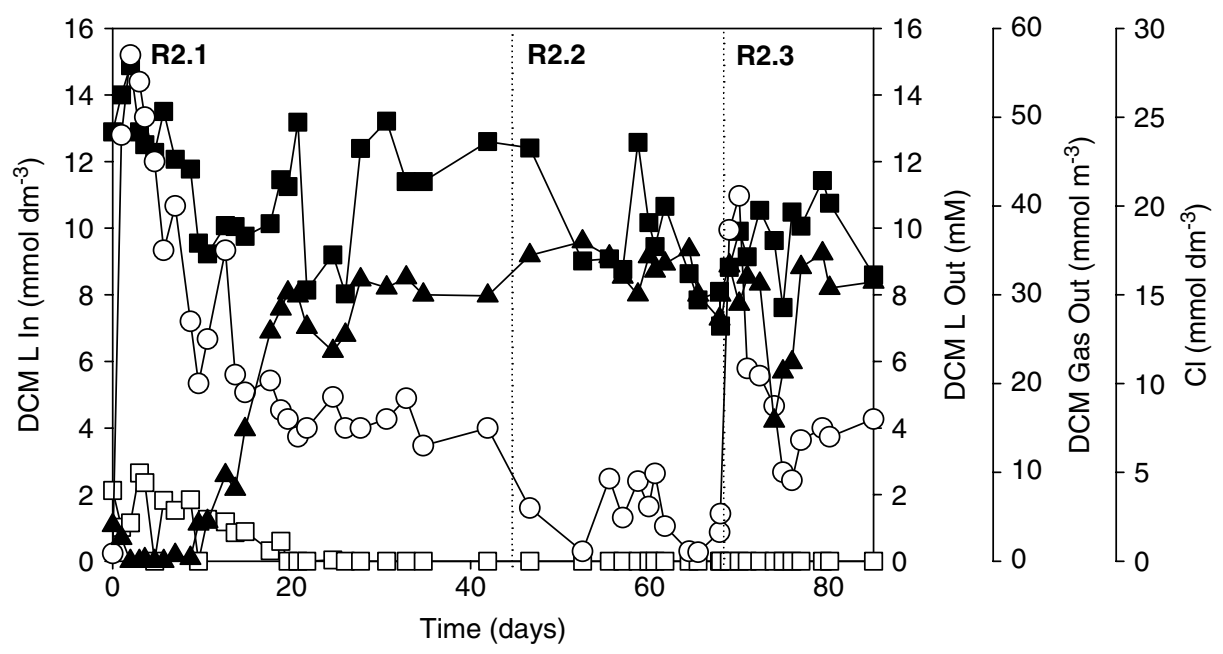

Figure 3. System R2. Symbols: DCM liquid in ( $\square$ ); DCM liquid out ( $\square$ ); DCM gas out (O); and $\mathrm{Cl}^{-}(\boldsymbol{\Delta})$. 
gaseous outlets, with the latter contributing to high emission levels. After day 10, the DCM measurable losses stabilized with negligible DCM concentrations observed at the liquid outlet. The biotrickling filter stabilized at a low treatment efficiency $(21 \%$ of the DCM fed) with a mean inlet concentration of $20 \mathrm{mmol} \mathrm{m}^{-3}$ and a specific elimination capacity of $176 \mathrm{mmol} \mathrm{m}^{-3}$ column $\mathrm{h}^{-1}$ upon achieving a pseudosteady state, given by constancy in the inlet gaseous stream observed after day 20 (Fig. 3). This resulted in an average DCM gaseous outlet concentration of $16 \mathrm{mmol} \mathrm{m}^{-3}$. As in System R1, low suspended biomass concentration in the CSTR was observed throughout the experiment.

A mass balance carried out during experiment R2.1 showed no significant differences in the BE (69\%), when compared with System R1 fed at the same conditions (Table 1), and an overall BEC of $11.6 \mathrm{~mol} \mathrm{~m}^{-3}$ system $\mathrm{d}^{-1}$. The measurable losses were decreased to $7 \%$ of the total DCM fed to the system, together with negligible DCM concentrations observed in the outlet liquid stream. However, the unmeasurable losses were increased to $24 \%$ of the total DCM. The contribution of the biotrickling filter was not relevant to the overall BEC (3\%) with a specific elimination capacity of $176 \mathrm{mmol} \mathrm{m}^{-3}$ column $\mathrm{h}^{-1}$. The relative importance between the BEC and the VR was increased to a factor of 10 from the decrease observed in the measurable losses.

Experiment R2.2 aimed at further decreasing the measurable losses of DCM by promoting recirculation of the outlet gaseous effluent from the CSTR through the reactor medium before entering the biotrickling column (Fig. 1). Recirculation was introduced on day 46 (Fig. 3). The DCM gaseous outlet concentration was decreased to an average of $4 \mathrm{mmol} \mathrm{m}^{-3}$ with a subsequent increase in the chloride concentration. Regarding the biotrickling filter, pseudo-steady state conditions were achieved after day 60 , with a treatment efficiency of $70 \%$ and a specific elimination capacity of $351 \mathrm{mmol} \mathrm{m}^{-3}$ column $\mathrm{h}^{-1}$.

A mass balance carried out during experiment R2.2 showed that recirculation of the CSTR headspace through the reactor medium increased the $\mathrm{BE}$ to $82 \%$ and the overall BEC to $12.2 \mathrm{~mol} \mathrm{~m}^{-3}$ system $\mathrm{d}^{-1}$ (Table 1 ). In addition, the measurable losses due to volatilization were reduced to $2 \%$ of the total DCM fed, with the biotrickling filter contributing $5 \%$ to the overall BEC. The unmeasurable losses were shown to be $16 \%$ of the total DCM. The higher treatment performance observed can be explained by the increase in the residence time of both liquid and gaseous streams achieved in the system due to recirculation. Overall, the BEC was 41 times higher than the VR.

Experiment R2.3 aimed at further investigating the recirculation effect by increasing it by a factor of 2 (day 68). An increase in DCM gaseous outlet concentration was immediately observed, concomitant with an initial decrease in chloride concentration. Due to the increase in the recirculating gaseous flow, the

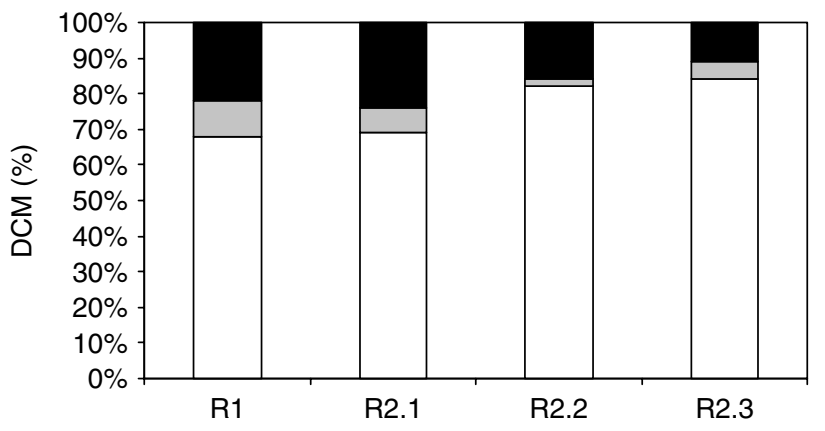

Figure 4. Overall comparison between the different operational strategies investigated. Symbols: DCM biodegraded ( $\square$ ); DCM measurable losses ( $($ ); and DCM unmeasurable losses ( $\square$ ).

DCM concentration at the inlet of the biotrickling filter was increased to $19 \mathrm{mmol} \mathrm{m}^{-3}$. However, the biotrickling filter showed similar specific elimination capacities to those observed during Experiment R2.2. Similar values of BEC were observed, as the chloride concentration stabilized at values similar to those observed in R2.2. Overall, no clear benefits were obtained by increasing the recirculation flow due to the increase in the DCM volatilization rate $(4.7 \%)$, while similar BECs (84\%) were achieved (Table 1 ). As a result of that increase, the ratio between the BEC and the VR was decreased to 17 .

Figure 4 summarizes the main results obtained in this study. When compared to the reference situation, Experiment R1, where a CSTR was operated for the treatment of a liquid stream containing DCM, the proposed operational strategies improved the overall treatment performance by successfully minimizing the volatilization losses. Recirculation of the gaseous effluent through the reactor medium was shown to be the most efficient strategy to improve the overall treatment performance (Experiment R2.2). However, when higher recirculation flows were used no further significant improvement in the BEC was observed (Experiment R2.3). The proposed biotrickling filter can be regarded as a polishing unit for abatement of any residual VOC present in the off gases.

\section{CONCLUSIONS}

Application of biological treatment in WWTPs handling chemical and pharmaceutical industry waste effluents is often difficult, mainly due to their high variability $^{16,17}$ and their volatility due to the presence of VOCs, such as DCM. ${ }^{11}$ Although the results obtained in Experiment R1 were comparable to other studies reported in the literature, the reactor design used in this work did not allow for the accumulation of high biomass concentration when compared to other designs (e.g. immobilized systems), thus limiting the achievement of high volumetric treatment performance. For this purpose, configurations that assure much higher biomass concentrations in the reactor, where biomass is immobilized, would be advisable. The major source of unmeasurable losses 
observed in this study was due to the pumping system used to maintain the liquid level constant in the CSTR. Overall, the chosen configuration allowed for identification of volatilization as the main source of measurable losses, which is in accordance with other studies that have shown volatilization to be the predominant source of VOCs emissions from WWTPs. ${ }^{3,4}$

This study introduced operational strategies for the aerobic biological treatment of effluents containing VOCs able to minimize losses due to volatilization, thus increasing the overall treatment efficiency. The ratio between the BEC and the VR was improved from 7 to 41 . Recirculation of the gaseous effluent through the reactor medium, which allowed for higher residence times, was shown to be a successful strategy for improving the treatment process, thus minimizing DCM volatilization losses. Given the wide range of sectors that are associated with the consumption and use of solvents containing VOCs, ${ }^{3}$ there is vast potential for application of the proposed strategies to control the emissions associated with the biological treatment of effluents containing VOCs in WWTPs.

\section{ACKNOWLEDGEMENTS}

This work was supported in part by the European Community's Human Potential Programme under contract HP-RTH-CT-2002-00213 (BIOSAP). R. Ferreira Jorge would like to acknowledge financial support from Fundação para a Ciência e a Tecnologia (SFRH/BPD/18716/2004).

\section{REFERENCES}

1 Bianchi AP and Varney MS, Volatilization processes in wastewater treatment plants as a source of potential exposure to VOCs. Ann Occup Hyg 41:437-454 (1997).

2 Keefe SH, Barber LB, Runkel RL and Ryan JN, Fate of volatile organic compounds in constructed wastewater treatment wetlands. Environ Sci Technol 38:2209-2216 (2004).

3 Atasoy E, Döğeroğlu T and Kara S, The estimation of NMVOC emissions from an urban-scale wastewater treatment plant. Water Res 38:3265-3274 (2004).
4 Nikolaou AD, Golfinopoulos SK, Kostopoulou MN, Kolokythas GA and Lekkas TD, Determination of volatile organic compounds in surface waters and treated wastewater in Greece. Water Res 36:2883-2890 (2001).

5 Easter C, Quigley C, Burrowes P, Witherspoon J and Apgar D, Odor and air emissions control using biotechnology for both collection and wastewater treatment systems. Chem Eng $\mathfrak{F}$ 113:93-104 (2005).

6 Leizinger T, Biodegradation of chlorinated aliphatic compounds. Current Opin Biotechnol 7:295-300 (1996).

7 Livingston AG, Extractive Membrane Bioreactors: a new process technology for detoxifying chemical industry wastewaters. f Chem Technol Biotechnol 60:117-124 (1994).

8 Pankhania M, Brindle K and Stephenson T, Membrane aeration bioreactors for wastewater treatment: completely mixed and plug-flow operation. Chem Eng F 73:131-136 (1999).

9 Brunner $\mathrm{W}$ and Leizinger $\mathrm{T}$, Bacterial degradation of dichloromethane. Experientia 34:1671-1675 (1978).

10 Herbst $\mathrm{B}$ and Wiesmann $\mathrm{U}$, Kinetics and reaction engineering aspects of the biodegradation of dichloromethane and dichloroethane. Water Res 30:1069-1076 (1996).

11 Klečka GM, Fate and effects of methylene chloride in activated sludge. Appl Environm Microbiol 44:701-707 (1982).

12 Bastos FSC, Castro PML and Ferreira Jorge RM, Biological treatment of a contaminated gaseous emission from a paint and varnish plant - from laboratory studies to pilot-scale operation. f Chem Technol Biotechnol 78:1201-1207 (2003).

13 Iwasaky I, Utsumi S, Hagino $\mathrm{K}$ and Owzawa T, A new spectrophotometric method for the determination of small amounts of chloride using the mercury thiocyanate method. $\mathcal{F}$ Chem Soc fpn 29:860-864 (1956).

14 Lamarche P and Droste RL, Air-Stripping mass transfer correlations for volatile organics. $\mathcal{J}$ Air Waste Manag Assoc 89:78-89 (1989).

15 Gälli R and Leizinger T, Specialized bacterial strains for the removal of dichloromethane from industrial waste. Conservation Recycle 8:91-100 (1985).

16 Stucki G, Biological decomposition of dichloromethane from a chemical process effluent. Biodegradation 1:221-228 (1990).

17 LaPara TM, Nakatsu CH, Pantea LM and Alleman JE, Phylogenetic analysis of bacterial communities in mesophilic and thermophilic bioreactors treating pharmaceutical wastewater. Appl Environm Microbiol 66:3951-3959 (2000).

18 LaPara TM, Nakatsu CH, Pantea LM and Alleman JE, Stability of the bacterial communities supported by a sevenstage biological process treating pharmaceutical wastewater as revealed by PCR-DGGE. Water Res 36:638-646 (2002). 